\title{
Double Stroke and Double Malignancy: A Case Report
}

\section{Kehaya $S^{*}$}

Department of Neurology, Trakya University Medical Faculty, Edirne, Turkey

${ }^{*}$ Corresponding author: Kehaya S, Department of Neurology, Trakya University Medical Faculty, Edirne, Turkey, E-mail: sezginkehaya@yahoo.com

Citation: Kehaya S (2017) Double Stroke and Double Malignancy: A Case Report. J Case Rep Stud 5(5): 501

Received Date: August 29, 2017 Accepted Date: October 25, 2017 Published Date: October 27, 2017

\begin{abstract}
Stroke associated with malignancy is rapidly increasing. Lesion types mainly resemble cardio embolic multiple ischemic lesions. We present a rare stroke patient with both ischemic and hemorrhagic lesions simultaneously. The etiology of stroke was found to be due to lung cancer and essential thrombocytosis. Differential diagnosis in this case can help for further investigation of strokes with malignancy.
\end{abstract}

Keywords: Stroke; Intracerebral hemorrhage; Myeloploriferative disease; Lung cancer

\section{Introduction}

Malignancy is a risk factor for ischemic stroke due to hypercoagulability. Lung cancer is the most prevalent etiology but other solid tumors and hematological malignancies have been associated too [1]. The lesions of ischemic stroke reported to be multiple acute and chronic ischemic lesions in multiple vascular territories [2]. Intracranial hemorrhage in cancer patients were observed due to metastatic intratumoral hemorrhage or coagulopathy. Hemorrhage was mainly reported in myeloproliferative neoplasm (MPN) [3]. For secondary prevention of ischemic stroke, antiaggregant or anticoagulant agents are used, which are contraindicated in brain hemorrhage. If both ischemia and hemorrhage is present, treatment option is a dilemma. We present a stroke case with ischemic and hemorrhagic lesions due to lung cancer and essential thrombocytemia. We think that the coexistence of 2 malignancies and 2 stroke types together in a case is very rare and deserve to be shared.

\section{Case Presentation}

Seventy four years old male patient referred to emergency department with sudden onset dysarthria and right hemiparesis. He was felt right sided numbness in his body after micturition in that morning. 10 minutes after that his right arm and leg become

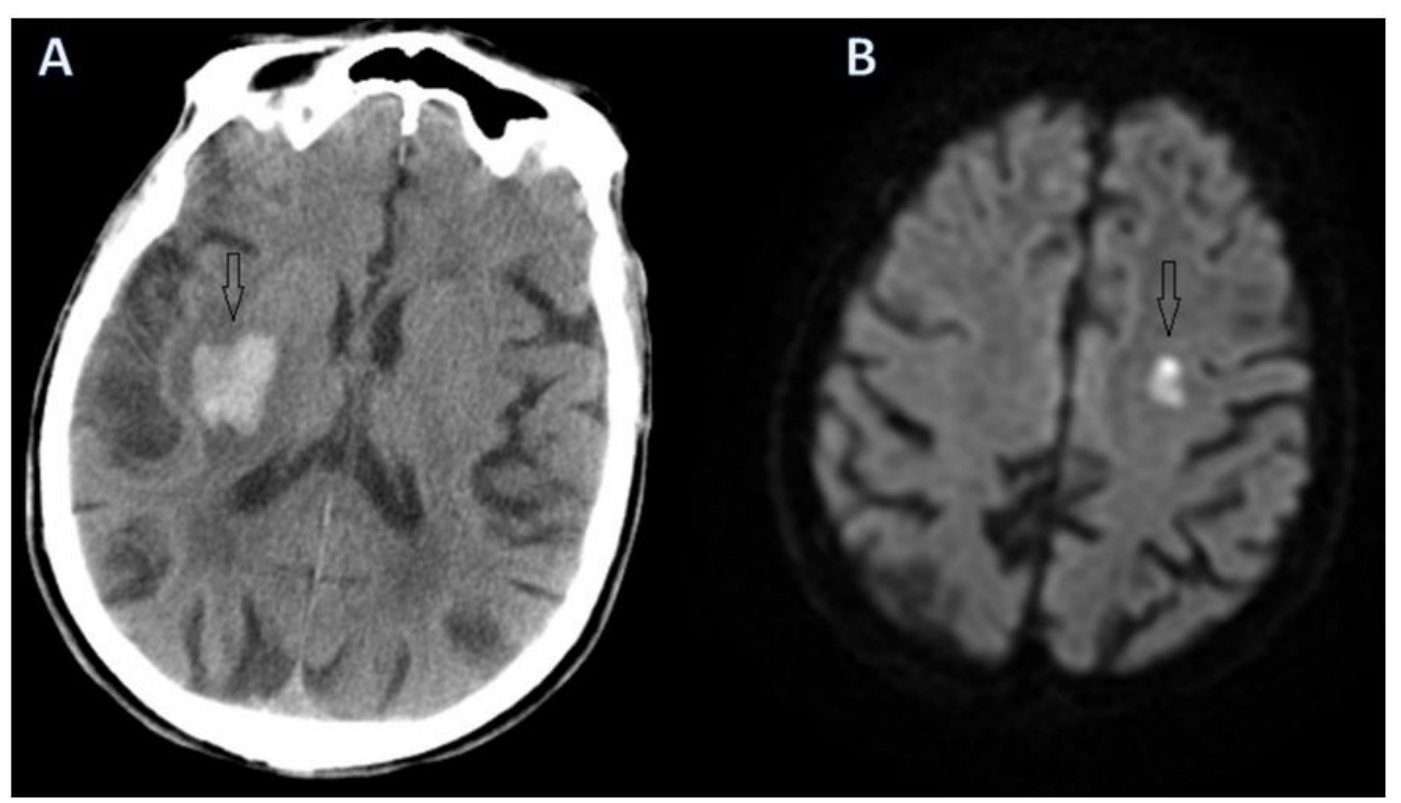

Figure 1: Brain CT and DWI-RMI. A: Brain CT shows hemorrhage in right basal ganglionic area. B: DWI-MRI shows acute ischemia 
week and his speech deteriorated. At his neurological examination, done at $5^{\text {th }}$ hour after symptom beginning, he was conscious, the speech was slurred but understandable, he was obeying complex orders, his cranial nerve examination revealed only slight right facial upper motor neuron weakness. He had right hemihipoesthesia, right hemiparesia of 4/5 muscle strength and bilateral positive Babinski sign. His systemic examination was unremarkable except rales in chest. His arterial tension was $130 / 80 \mathrm{~mm} \mathrm{Hg}$. 12-lead electrocardiography revealed no pathology. He was using only talmusoline for benign prostate hyperplasia. He was smoker and used 10 cigarettes per day since 50 years. The plain chest X-ray revealed hilar enlargement and emphysematous changes. At brain computerized tomography (CT) we observed right basal ganglionic hematoma incompatible with the clinical symptoms. Diffusion Weighted Magnetic Resonance Images (DWI-MRI) showed frontal subcortical lacunar ischemic lesion at left corona radiata level (Figure 1).

In laboratory workup blood tests were: urea $27 \mathrm{mg} / \mathrm{dl}$, creatinin $1 \mathrm{mg} / \mathrm{dl}$, ALT $8 \mathrm{U} / \mathrm{L}$, AST $27 \mathrm{U} / \mathrm{L}$, LDH $499 \mathrm{U} / \mathrm{L}$, serum glucose $85 \mathrm{mg} / \mathrm{dl}$, C-reactive protein $4.14 \mathrm{mg} / \mathrm{dl}$, prothrombine time $15.4 \mathrm{sec}$, INR 1.18, WBC 11.5 X10^3 uL, RBC $6.7 \mathrm{X} 10^{\wedge} 6 \mathrm{uL}, \mathrm{Hgb}$ $12 \mathrm{gr} / \mathrm{dl}, \mathrm{MCV} 60.9 \mathrm{fL}, \mathrm{HCT}$ 40.8\%, Plt 1084 X10^3 uL, RDW-CV 19.2. Hematologic consultation declared contraindication for antiaggregant treatment because of thrombocytosis and advised screening for reactive thrombocytosis or MPN. The patient was hospitalized at neurology service for follow up. He received only intravenous fluid replacement for treatment. At $3^{\text {rd }}$ day of hospitalization he had become difficulty in breathing. At his arterial blood gas examination $\mathrm{pH}: 7.36, \mathrm{PO}_{2}: 41.6 \mathrm{mmHg}, \mathrm{PCO}_{2}: 48.6$ $\mathrm{mmHg}$, SO2:76.8 were found. Pulmonary medicine consultation advised oxygen supplement only and asked for thorax CT, serum ACE level, cranium and wrist X-ray for sarcoidosis. Serum ACE level, calcium level, cranium and wrist X-rays were normal but thorax CT revealed mediastinal pulmonary mass lesion with plevral and costal invasion. 18 Flurodeoxyglucose (FDG) Positron Emission CT was revealed left pulmonary lesion with hilar lymph node and spinal bone metastasis (Figure 2). There was splenomegaly but no increased FDG in spleen. The patient was regarded as lung cancer. Adenocarcinoma or malign mesothelioma was considered for differential diagnosis and pleural biopsy was advised for pathologic diagnosis.

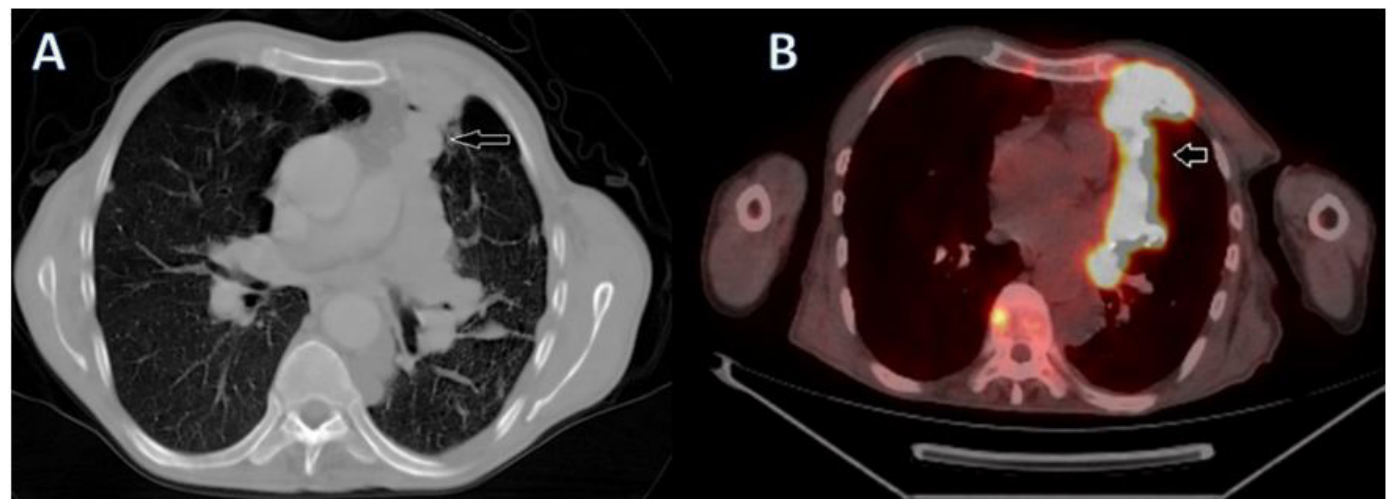

Figure 2: Thorax CT and PET-CT. A: Thorax CT shows mass lesion. B: PET-CT shows hyper-metabolic mass lesion with pleural and costal invasion

Cranial MRI showed periventricular chronic ischemic lesions, right basal ganglionic hemorrhage and acute left corona radiata lacunar ischemia. Cranial and cervical MR Angiography revealed some atherosclerotic changes but there was no meaningful narrowing of arterial structure. There was no pathological contrast enhancement in hemorrhagic area in gadolinium MRI. Transthoracic echocardiography showed type 1 left ventricular dysfunction and no thrombosis or vegetation on valves. There was no hyperlipidemia or hyperglycemia. Hematological tests revealed fibrinogen $390 \mathrm{mg} / \mathrm{dl}$, Di-dimer $0.76 \mu \mathrm{g} / \mathrm{mL}$, ferritin 9.9 $\mathrm{ng} / \mathrm{mL}$, serum iron $9 \mathrm{ug} / \mathrm{dl}$, total iron binding capacity $260 \mathrm{ug} / \mathrm{dl}$, BCR/ABL t(9;22) FISH was negative. Real Time PCR JAK-2 V617F mutation analyses was found over $5 \%$ positive. After 6 days of hydration Plt decreased to $721 \mathrm{X} 10^{\wedge} 3 \mathrm{uL}$. These findings were consisted with essential thrombocytosis. Control CT showed regression of right basal ganglionic hematoma. At $10^{\text {th }}$ day of admission the patient had severe dyspnea and because of hypoxia he was transferred for assisted ventilation to intensive care unit. We could not obtain pleural biopsy for diagnosis of lung cancer and he died 7 days after. We regarded the etiology of ischemic stroke as diathesis for thrombosis because of malignancy and hemorrhagic stroke etiology because of bleeding diathesis in thrombocytosis.

\section{Discussion}

The cause of right hemiparesia of the patient was the left corona radiata ischemic lesion and the right basal ganglionic hematoma was asymptomatic. Simultaneous intra cerebral hematoma and ischemia in our patient could be explained by thrombocytosis. Intra cerebral basal ganglionic hematoma mainly occurs in case of hypertension and rarely due to other causes such as cerebral amyloid angiopathy, bleeding diathesis, hemorrhagic transformation of ischemia and drug related causes [4]. The patient was normotensive and brain MRI showed no signs of angiopathy. For etiologic explanation of ischemia we excluded cardioembolic sources by echocardiography and electrocardiograpy. With blood tests, MRI and MRI Angiography we excluded large atherosclerosis and small vessel disease, in which vascular risk factors such as diabetes and dyslipidemia was blamed. Because the patient had platelet count over $1000 \times 10^{\wedge} 3 \mathrm{~mm}^{\wedge} 3$ and JAK-2 mutation were positive consisted with essential thrombocytosis (ET), we regarded the stroke etiology as other known causes. After diagnosis of lung cancer the issue had changed. Malignancy could cause thrombosis and bleeding, too. 
The mechanism of stroke in malignancy is mainly associated with disseminated intravascular coagulation (DIC), in which thrombosis occurs due to thrombi in vessels and bleeding due to exhausted coagulation factors [3]. Although Di-dimer level of our patient was normal and there was no clinic of DIC, because two possible explanations could cause the ischemic stroke, the diagnosis changed to unknown etiology. The basal ganglionic hemorrhage could be caused by metastasis. But there was no atypical contrast enhancement in our MRI and no hypermetabolism in PET CT. Besides, on control brain CT the hemorrhage was regressed. That's why we excluded the metastasis and decided coagulopathy for hemorrhagic etiology. MRI lesions in stroke patients with malignancy were investigated and multiple lesions in multiple vascular territories, acute and chronic multiple ischemic lesions were reported [2]. But we couldn't find studies for significance of ischemic and hemorrhagic lesions together. Septic emboli could be thought for differential diagnosis and in our patient there was no any sign for vegetation in echocardiography.

Although ET diagnosis is an exclusion diagnosis of other MPN and no other malignancy must be present, we regarded our patient as ET, because only thrombocytes were elevated in follow up and erythrocytes, leucocytes were normal. Lung cancer could cause reactive thrombocytosis but JAK-2 V617F mutation was associated mainly with MPN [5]. If we could completed the work up for MPN the diagnosis could be changed to other Philadelphia negative MPN such as polycythemia vera or myelofibrosis. Nevertheless, there would be two different malignancies, lung cancer and MPN. It was showed that in time, myeloid neoplasm can shift to one another and in a recent case report with JAK2 V617F mutation, lung adenocarcinoma, leukemia and lymphoma was reported in the same patient [6]. Association between solid, myeloploriferative and lymphoploriferative neoplasm had been questioned in JAK-2 V617F mutation. In another old experimental study diffuse malignant mesothelioma cells were successfully treated with interferon gamma and this was explained by JAK-STAT pathway, which may be related with solid tumors and MPN in case of JAK2 mutation as ours [7]. JAK2 was related with metastasis in metastatic lung cancer, too [8]. Thrombosis diathesis in ET was widely investigated and increased age, cardiovascular risk factors, previous thrombosis history and JAK2 V617F mutation were correlated [9]. As possible pathophysiology for thrombosis, increased viscosity, marginalization of thrombocytes to vascular endothelium and hypercoagulability had been proposed. Thrombocyte count however was inversely associated with thrombosis. Patients with low platelet count had more thrombosis risk and patients with high platelets low risk, but an increased risk for bleeding [10]. Bleeding also was associated linearly with thrombocyte count. Acetylsalicylic acid had been shown to decrease the risk of thrombosis in ET but can increase bleeding in high platelet count. Treatment option in such a case was proposed to decrease platelet count with hydoxyurea first and use of acetylsalicylic acid thereafter [5]. JAK2 V617F mutation can be of value not only for association with thrombosis but also with high mortality in MPN and in stroke patients.

\section{Conclusion}

As a conclusion, if hemorrhagic and ischemic lesions are found in a patient, malignancy can be suspected. In case of hematologic malignancy in older age consideration for other prevalent malignancies must be kept in mind.

\section{References}

1. Selvik HA, Thomassen L, Bjerkreim AT, Næss H (2015) Cancer-Associated Stroke: The Bergen NORSTROKE Study. Cerebrovasc Dis Extra 5: 107-13.

2. Schwarzbach CJ, Fatar M, Eisele P, Ebert AD, Hennerici MG, et al. (2015) DWI Lesion Patterns in Cancer-Related Stroke - Specifying the Phenotype. Cerebrovasc Dis Extra 5: 139-45.

3. Velander AJ, DeAngelis LM, Navi BB (2012) Intracranial hemorrhage in patients with cancer. Curr Atheroscler Rep 14: 373-81.

4. Simon RP, Aminoff MJ, Greenberg DA (2009) Clinical Neurology (7 $7^{\text {th }}$ Edn) McGraw-Hill New York, United States 318-24.

5. Artoni A, Bucciarelli P, Martinelli I (2014) Cerebral thrombosis and myeloproliferative neoplasms. Curr Neurol Neurosci Rep 14: 496.

6. Liu KG, Verma A, Derman O, Kornblum N, Janakiram M, et al. (2016) JAK2 V617F mutation, multiple hematologic and non-hematologic processes: an association? Biomarker Res 4: 19.

7. Buard A, Vivo C, Monnet I, Boutin C, Pilatte Y, et al. (1998) Human malignant mesothelioma cell growth: activation of janus kinase 2 and signal transducer and activator of transcription 1alpha for inhibition by interferon-gamma. Cancer Res 58: 840-7.

8. Wang P, Lv HY, Zhou DM, Zhang EN (2016) miR-204 suppresses non-small-cell lung carcinoma (NSCLC) invasion and migration by targeting JAK2. Genet Mol Res 15

9. Barbui T, Finazzi G, Carobbio A, Thiele J, Passamonti F, et al. (2012) Development and validation of an International Prognostic Score of thrombosis in World Health Organization-essential thrombocythemia (IPSET-thrombosis). Blood 120: 5128-33.

10. Carobbio A, Finazzi G, Antonioli E, Guglielmelli P, Vannucchi AM, et al. (2008) Thrombocytosis and leukocytosis interaction in vascular complications of essential thrombocythemia. Blood 112: 3135-7. 


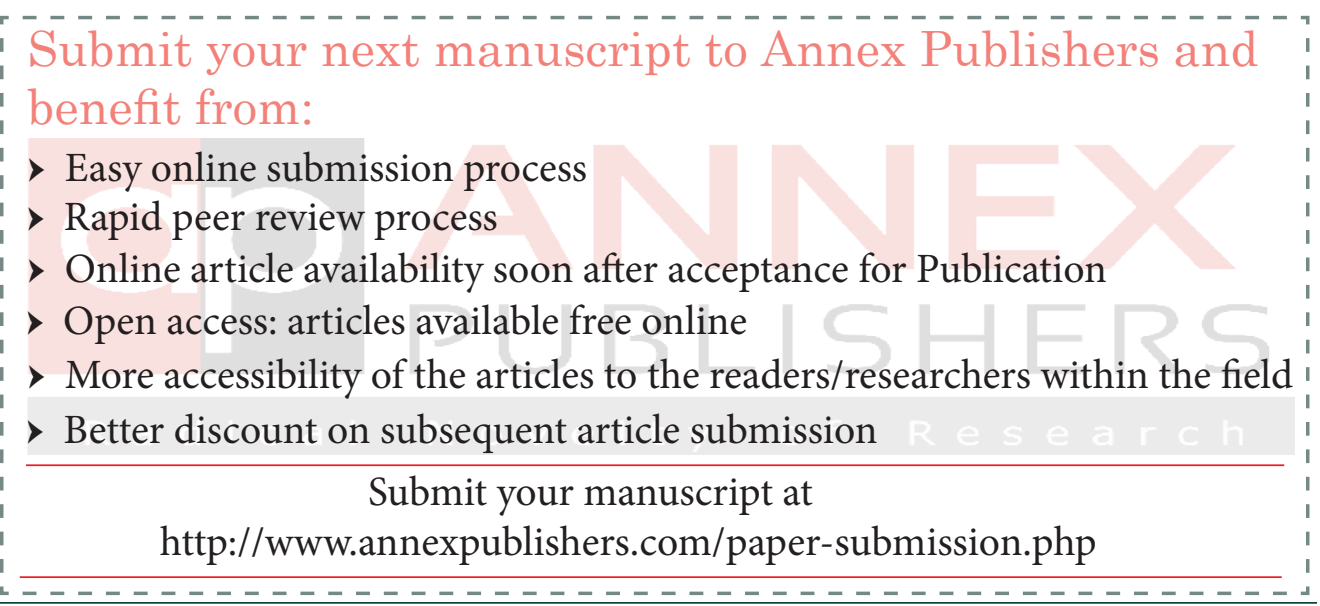

